\title{
Remote magnetic versus manual catheter navigation for circumferential pulmonary vein ablation in patients with atrial fibrillation
}

\author{
Lars Lüthje · Dirk Vollmann · Joachim Seegers • \\ Marc Dorenkamp • Christian Sohns • \\ Gerd Hasenfuss · Markus Zabel
}

Received: 9 February 2011/ Accepted: 8 June 2011/Published online: 25 June 2011

(C) The Author(s) 2011. This article is published with open access at Springerlink.com

\begin{abstract}
Background Only limited data exist on the clinical utility of remote magnetic navigation (RMN) for pulmonary vein (PV) ablation. Aim of this prospective study was to evaluate the safety and efficacy of RMN for PV isolation as compared to the manual (CON) approach.

Methods and results A total of 161 consecutive patients undergoing circumferential PV isolation were included. Open-irrigated $3.5 \mathrm{~mm}$ ablation catheters under the guidance of a mapping system were used. The catheter was navigated with the Stereotaxis Niobe II system in the RMN group $(n=107)$ and guided manually in the CON group $(n=54)$. Electrical isolation of all PVs was achieved in $90 \%$ of the patients in the RMN group and in $87 \%$ in the CON group $(p=0.6)$. All subjects were followed every 3 months by $7 \mathrm{~d}$ Holter-ECG. At 12 months of follow-up, $53.5 \%(\mathrm{RMN})$ and $55.5 \%(\mathrm{CON})$ of the patients were free of any left atrial tachycardia/atrial fibrillation (AF) episode $(p=0.57)$. Free of symptomatic AF recurrence were $66.3 \%(\mathrm{RMN})$ and $62.1 \%(\mathrm{CON})$ of the subjects $(p=0.80)$. Use of RMN was associated with longer procedure duration $(p<0.0001)$, ablation times $(p<0.0001)$, and RF current application duration $(p<0.05)$. In contrast, fluoroscopy time was lower in the RMN group $(p<0.0001)$. Major complications occurred in 6 of 161 procedures $(3.7 \%)$, with no significant difference between groups $(p=0.75)$.
\end{abstract}

L. Lüthje and D. Vollmann contributed equally to this work.

L. Lüthje $(\bowtie) \cdot$ D. Vollmann · J. Seegers · M. Dorenkamp · C. Sohns - G. Hasenfuss - M. Zabel

Division of Cardiology and Pulmology, Heart Center, University of Göttingen, Robert-Koch-Str. 40, 37075 Göttingen, Germany e-mail: larsluethje@med.uni-goettingen.de
Conclusion RMN-guided PV ablation provides comparable acute and long-term success rates as compared to manual navigation. Procedural complication rates are similar. The use of RMN is associated with markedly reduced fluoroscopy time, but prolonged ablation and procedure duration.

Keywords Ablation - Electrophysiology ·

Atrial fibrillation · Pulmonary vein ablation .

Remote magnetic navigation

\section{Introduction}

In the past years, ablation strategies to isolate the pulmonary veins have become an established treatment choice for patients with drug-refractory symptomatic atrial fibrillation (AF) [1]. Navigating the catheter safely within the left atrium to produce effective ablation lesions at all desired locations can prove to be challenging even for the skilled electrophysiologist. Long-term success rates of the procedure, defined by freedom from AF during follow-up, range between 60 and $80 \%$ [2], whereas severe complications may occur in up to $4.5 \%$ of the cases [3]. Stiff ablation catheters mandate frequent visualization of the catheter tip during manipulation, therefore, procedure related radiation exposure may be extensive to patient and operator [4]. Efforts continue to improve the safety and efficacy of this widely applied procedure.

Recently, a magnetic catheter navigation system (Niobe II, Stereotaxis Inc., St. Louis, MO, USA) for remote mapping and ablation of cardiac arrhythmias has been introduced. Potential benefits of this system comprise a reduction of radiation exposure as well as improved procedural safety and efficacy due to an unrestricted and more 
precise movement of the soft catheter tip. So far, only limited data are available on the exact clinical utility of the remote magnetic catheter navigation (RMN) system for pulmonary vein $(\mathrm{PV})$ isolation and treatment of AF [5-10].

The aim of this prospective observational study was to evaluate the safety and efficacy of the RMN-system for PV isolation and treatment of $\mathrm{AF}$ as compared to a conventional manual approach.

\section{Methods}

Patient selection

Consecutive patients undergoing circumferential PV isolation at our centre between April 2008 and April 2010 were included in this study. All patients had conventional indications with symptomatic AF and a failed attempt to maintain sinus rhythm with antiarrhythmic medication [1]. Conventional manual AF ablation was introduced at our center in 2006. The RMN-system was utilized for all AF ablation procedures beginning November 2008. The control group consisted of those 54 consecutive patients that underwent conventional manual ablation (CON) between April and October 2008 (before switching to RMN-guided ablation).

Exclusion criteria were hyperthyroidism, LA thrombus, decompensated heart failure, stroke, myocardial infarction or gastrointestinal bleeding within 4 weeks prior to the intervention, and life-expectancy $<6$ months.

\section{Electrophysiological study}

In all subjects, left atrial (LA) thrombi were excluded by transesophageal echocardiography, and LA anatomy was acquired by high-resolution thoracic computer tomography imaging just prior to the procedure. All ablation procedures were performed during conscious sedation using intravenous sufentanil, midazolam and/or propofol under continuous monitoring of blood pressure and oxygen saturation.

For the electrophysiological procedure, all catheters were advanced via the femoral vein. A 6F steerable decapolar catheter (Bard Dynamic Tip, Bard Inc., Lowell, MA, USA) was positioned in the coronary sinus. After a fluoroscopically guided transseptal puncture an SL1 sheath (St. Jude Medical, Inc., St. Paul, MN, USA) in the RMN group or an Agilis deflectable sheath (St Jude Medical) in the CON group were advanced into the LA. Intravenous heparin was administered immediately after the transseptal puncture to maintain an activated clotting time of 250-300 s throughout the procedure. In the RMN group, a $3.5 \mathrm{~mm}$ open-irrigated, magnetic mapping and ablation catheter (Navistar Thermocool RMT, Biosense Webster,
Diamond Bar, USA) was advanced through the sheath into the LA, whereas in the CON group a manually guided $3.5 \mathrm{~mm}$ open-irrigated mapping and ablation catheter (Navistar Thermocool, Biosense Webster) was used. Patients presenting with sustained atrial fibrillation underwent electrical cardioversion for mapping and ablation.

Circumferential pulmonary vein ablation was performed using a three-dimensional mapping system (CartoMerge $\mathrm{XP}$, Biosense Webster) in conjunction with the integrated CT image of the LA and real-time fluoroscopy. In the RMN group, the Niobe II magnetic navigation system (Stereotaxis) and a joystick-controlled motor drive (Cardiodrive, Stereotaxis) were utilized for remote magnetic navigation of the ablation catheter, whereas in the CON group the ablation catheter was guided manually.

The RMN system has been described in detail elsewhere [10]. Briefly, two permanent magnets located on either side of the procedure desk generate a magnetic field $(0.08$ Tesla) within the patient. The magnetic ablation catheter incorporates four magnets in the distal portion of the catheter. A change of the desired vector for catheter orientation on a computer screen results in alteration of the magnetic field generated by the permanent magnets and thereby corresponding deflection of the magnetic catheter within the heart. The joystick-controlled motor drive allows catheter advancement and retraction. Thus, the system provides complete remote catheter navigation for mapping and ablation.

The radiofrequency $(\mathrm{RF})$ generator (Stockert, Biosense Webster) was set to temperature controlled RF delivery with a target temperature of $45^{\circ} \mathrm{C}$ and a nominal power limit of $40 \mathrm{~W}$ (flow $30 \mathrm{ml} / \mathrm{min}$ ) and $30 \mathrm{~W}$ (flow $17 \mathrm{ml} /$ $\min$ ) at the posterior LA wall. RF current was applied for 30-60 s until local electrogram amplitude was reduced by $80 \%$. Endpoint of the ablation procedure was the electrical isolation of all PVs defined as bidirectional conduction block. This was verified by careful and repeated mapping for residual potentials around the entire circumference of the PV ostia, and pacing from multiple sites within the circumferential line.

Follow-up

All patients were monitored in the hospital at least overnight. Echocardiography was performed within $24 \mathrm{~h}$ after the procedure and in cases of unexplained hypotension to rule out pericardial effusion. On the day after the procedure, the venous puncture site was inspected for significant local hemorrhage, and a 12-lead surface ECG was acquired to confirm normal sinus rhythm. Antiarrhythmic medication was administered according to the investigators discretion. Oral anticoagulation was restarted the day after the procedure with a target INR of 2.0-3.0. Bridging with 
unfractionated or low molecular weight heparin was initiated 6-12 h after the procedure. After hospital discharge, all patients were followed in our outpatient clinic every 3 months for at least 1 year. At each visit, subjects were asked for symptoms, documented arrhythmia recurrences, and current medication was assessed. Ambulatory holter monitoring was performed for 7 days at each follow-up to reveal possible arrhythmia recurrences. Furthermore, all patients were advised to present immediately in case of symptoms suggestive for arrhythmia recurrence and obtain ECG documentation. A documented AF or left atrial tachycardia (AT) episode lasting longer than 30 s outside a blanking period of 2 months after the index procedure were considered as recurrent AT/AF. Additional diagnostic information (e.g., echocardiogram, chest X-ray/computertomography) was acquired if symptoms were suggestive of procedure-related complications (e.g., pericardial effusion, pulmonary vein stenosis).

\section{Study endpoints}

The primary endpoint of this prospective observational study was acute procedural success, defined as the number of PVs isolated at the end of the procedure. Secondary endpoints were long-term procedural success, defined as long-term freedom from any AT/AF episodes irrespective of symptoms after the index procedure during 12 months of follow-up, and survival without symptomatic AF recurrence after the index procedure during 12 months of follow-up. Further secondary endpoints were freedom from symptomatic AF recurrence at the end of the individual observation period after the last PV ablation procedure irrespective of the number of PV ablations combined for both groups, procedure duration, ablation duration (defined as time from the first to the last ablation point), RF current application duration, fluoroscopy time, and procedure-related complications. Procedure-related complications were defined as death, atrio-esophageal fistulae, pulmonary vein stenosis requiring interventions, pericardial tamponade requiring drainage, systemic embolic events, phrenic nerve paralysis, femoral vessel damage requiring surgery, blood transfusion or prolongation of hospitalisation, and clinically and radiologically verified infections.

\section{Statistical analysis}

Continuous variables are expressed as mean \pm standard deviation or as median with inter-quartile range if appropriate. Normally distributed data were compared using the independent Student's $t$ test. Otherwise, comparisons between groups were performed using the Mann-Whitney $U$ test. One-way ANOVA or Kruskal-Wallis ANOVA (with Dunns post hoc test) were used to test for learning curve effects. A Kaplan-Meier analysis with the log-rank test was used to determine the probability of freedom from recurrent AT/AF. All tests are two-tailed. A $p$ value $<0.05$ is considered statistically significant.

We expected a mean acute $\mathrm{PV}$ isolation rate of $3.5 \pm 0.6 \mathrm{PVs}$ per patient. Based on this assumption a sample size of $100 \mathrm{RMN}$ and 50 control patients would allow detection of a difference in acute PV isolation of 0.3 PVs with $80 \%$ power and a two-tailed alpha of $5 \%$.

\section{Results}

Patient characteristics

A total of 161 patients were included. Data from 107 patients in the RMN group and 54 patients in the CON group were analysed. Patient baseline characteristics are illustrated in Table 1 and were comparable between groups. In both groups, about two-thirds of the patients were in persistent $\mathrm{AF}$, defined as $\mathrm{AF}$ lasting $>7$ days or requiring cardioversion according to current guidelines [11]. The persistent AF cohort included three patients with long-standing persistent AF (lasting for $\geq 1$ year) in the $\mathrm{CON}$ group and two in the RMN group. In both groups, $22 \%$ of the patients had a prior circumferential pulmonary vein ablation before the index procedure.

\section{Acute and long-term success}

At the end of the index procedure $3.7 \pm 0.8 \mathrm{PVs}$ in the CON group and $3.8 \pm 0.7 \mathrm{PVs}$ in the RMN group were isolated $(p=0.34)$. Electrical isolation of all PVs was achieved in $90 \%$ of the RMN-guided cases and in $87 \%$ of the subjects in the CON group ( $p=0.6)$.

By Kaplan-Meier analysis, the percentage of patients who were free of any AT/AF episode at 12 months of follow-up was $53.5 \%$ in the RMN group and $55.5 \%$ in the CON group ( $p=0.57$, Fig. 1). As illustrated in Fig. 2, the percentage of patients who were free of symptomatic AF recurrence at 12 months of follow-up was $66.3 \%$ in the RMN group and $62.1 \%$ in the CON group $(p=0.80)$. Antiarrhythmic drugs were prescribed to $25 \%$ of the patients without symptomatic AF recurrence in the $\mathrm{CON}$ group ( $n=32$ ) and to $29 \%$ in the RMN group $(n=36)$. Of those patients, $10 \%$ in the CON group and $11 \%$ in the RMN group were on amiodarone.

In both the RMN and CON group, long-term success rates (as defined by freedom from AT/AF during followup) tended to be higher in patients with a history of paroxysmal versus persistent AF (RMN: 66 vs. 49\%; CON: 63 vs. $52 \%$ ) and in patients with LA diameter $\leq 45 \mathrm{~mm}$ as 
Table 1 Patient Characteristics

\begin{tabular}{llll}
\hline & RMN $(n=107)$ & CON $(n=54)$ & $P$ value \\
\hline Gender (male/female) & $66 / 41$ & $34 / 20$ & $\mathrm{~ns}$ \\
Age (years) & $62 \pm 10$ & $61 \pm 10$ & $\mathrm{~ns}$ \\
Arterial hypertension & $75(70 \%)$ & $40(74 \%)$ & $\mathrm{ns}$ \\
Coronary artery disease & $24(22 \%)$ & $7(13 \%)$ & $\mathrm{ns}$ \\
Hypertensive heart disease & $27(25 \%)$ & $7(13 \%)$ & $\mathrm{ns}$ \\
Other & $19(17 \%)$ & $5(11 \%)$ & $\mathrm{ns}$ \\
Lone AF & $14(13 \%)$ & $7(13 \%)$ & $\mathrm{ns}$ \\
LA size (mm) & $47 \pm 6$ & $45 \pm 7$ & $\mathrm{~ns}$ \\
LVEF (\%) & $55 \pm 8$ & $55 \pm 8$ & $\mathrm{~ns}$ \\
Paroxysmal AF & $34(32 \%)$ & $18(33 \%)$ & $\mathrm{ns}$ \\
Persistent AF & $73(68 \%)$ & $36(67 \%)$ & $\mathrm{ns}$ \\
Prior AF ablation & $23(22 \%)$ & $12(22 \%)$ & $\mathrm{ns}$ \\
Previous AADs & $1.1 \pm 0.8$ & $1.4 \pm 0.8$ & $\mathrm{~ns}$ \\
Flecainide & $54(50 \%)$ & $34(63 \%)$ & $\mathrm{ns}$ \\
Propafenone & $12(11 \%)$ & $4(6 \%)$ & $\mathrm{ns}$ \\
Sotalol & $14(13 \%)$ & $12(22 \%)$ & $\mathrm{ns}$ \\
Amiodarone & $40(37 \%)$ & $19(37 \%)$ & $\mathrm{ns}$ \\
\hline
\end{tabular}

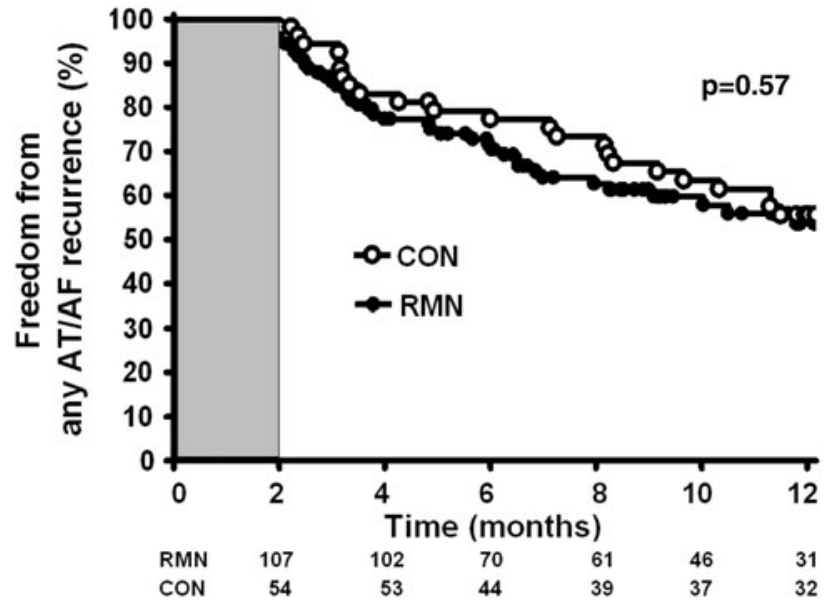

Fig. 1 Freedom from any AT/AF recurrence in the CON group versus the RMN group. The Kaplan-Meier analysis during the first 12 months of follow-up revealed no significant difference between the two groups

compared to LA > $45 \mathrm{~mm}$ (RMN: 67 vs. $44 \%$; CON: 64 vs. $47 \%$ ) (Fig. 3).

Freedom from symptomatic AF combining both groups after a follow-up duration of $382 \pm 187$ days and $1.5 \pm 0.6 \mathrm{PV}$ isolations (including PV isolations before the index procedure) was $83 \%$. Following the index procedure, additional PV ablation procedures were considered only outside the blanking period and were performed in $23 \%$ of the patients in the RMN group and in $24 \%$ of the patients in the CON group. A total of $40 \%$ of these patients were on antiarrhythmic drugs at the end of

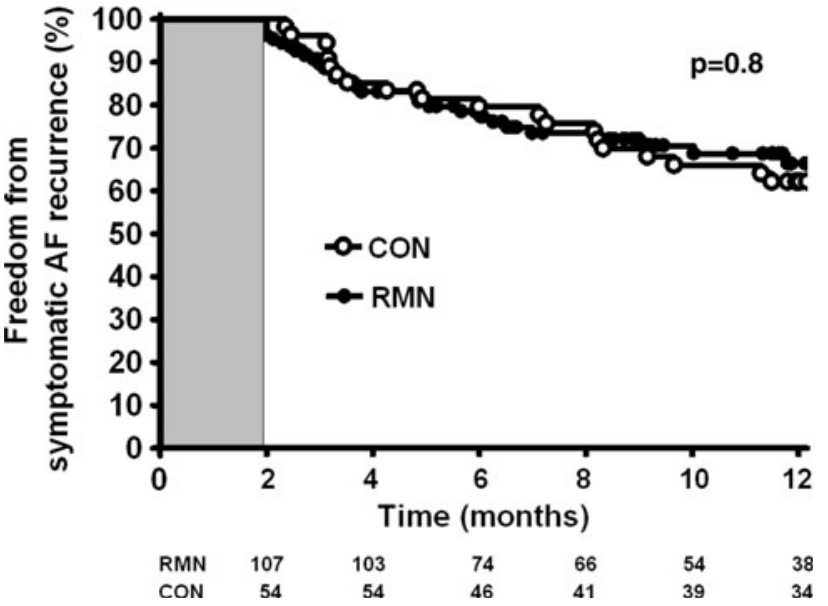

Fig. 2 Freedom from symptomatic AF recurrence in the CON group versus the RMN group. The Kaplan-Meier analysis during the first 12 months of follow-up revealed no significant difference between the two groups

the respective observation period with $14 \%$ of the patients being on amiodarone.

Procedural parameters and complications

As illustrated in Fig. 4, use of the RMN system was associated with longer procedure duration (RMN: $225.5 \pm 54.6 \mathrm{~min}, \mathrm{CON}: 165.6 \pm 52.4 \mathrm{~min}, p<0.0001)$, longer ablation times (RMN: $125.3 \pm 46.5 \mathrm{~min}, \mathrm{CON}$ : $79.6 \pm 28.5 \mathrm{~min}, p<0.0001)$ and longer RF current application duration (RMN: $50.4 \pm 17.7 \mathrm{~min}, \mathrm{CON}$ : 

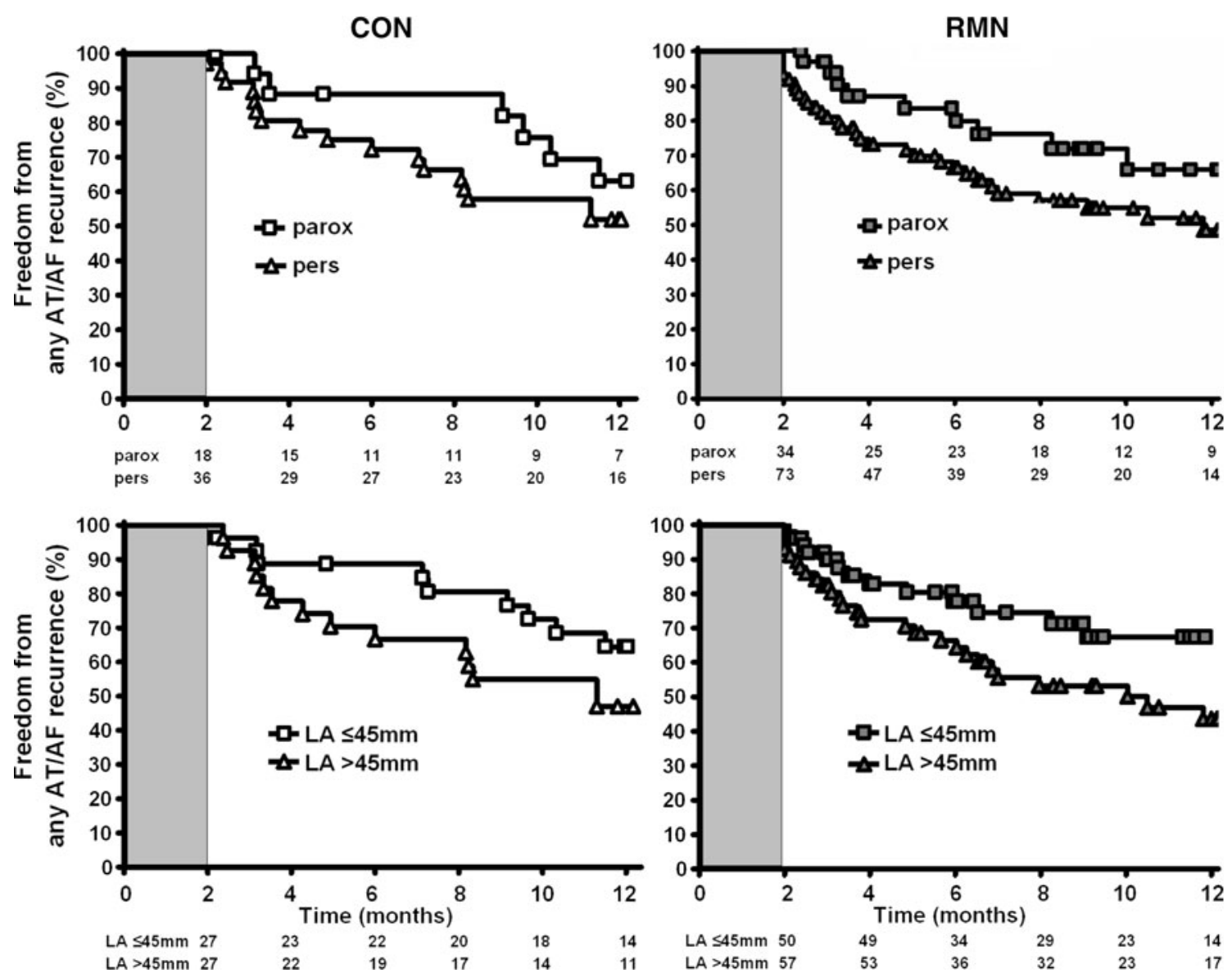

Fig. 3 Freedom from any AT/AF recurrence dependent on type of AF upon presentation for initial procedure (paroxysmal vs. persistent, upper panels) and LA size (lower panels). In both the RMN (right panels) and CON (left panels) group, long-term success rates tended

$43.9 \pm 11.0 \mathrm{~min}, p<0.05)$ as compared to the $\mathrm{CON}$ group. In contrast, fluoroscopy time was lower in the RMN [12 (9-17) min] than in the CON [37 (29-44) min] group $(\mathrm{p}<0.0001)$. Furthermore, fluoroscopy time significantly decreased in the RMN group over time (Fig. 5). No learning curve effect was observed for the remaining procedural parameters (procedure duration, ablation time and RF current application duration).

Major complications occurred in 6 of the 161 procedures $(3.7 \%)$, with no significant difference between the 2 treatment groups $(p=0.75)$. One procedure-related complication was observed in the $\mathrm{CON}$ group (aspiration pneumonia). In the RMN group, procedure-related complications were cardiac tamponade requiring pericardial puncture $(n=2)$, hematoma after femoral vein puncture requiring blood transfusion $(n=1)$ or prolonging hospital stay $(n=1)$, and transient ischemic attack $(n=1)$. Cardiac tamponades occurred subacutely, in one case $30 \mathrm{~min}$ after the end of an uneventful procedure, in the other subject several hours after an uncomplicated ablation. to be higher in patients with a history of paroxysmal AF than in subjects with persistent $\mathrm{AF}$ and in patients with an LA diameter $\leq 45 \mathrm{~mm}$ as compared to $\mathrm{LA}>45 \mathrm{~mm}$

\section{Discussion}

Main finding

The main finding of this prospective observational study is that RMN-guided circumferential PV ablation with an open-irrigated mapping and ablation catheter results in comparable acute and long-term success and complication rates as compared to the $\mathrm{CON}$ approach. Utilization of the RMN system reduces fluoroscopy time, but increases ablation and total procedure times as compared to the CON ablation technique.

Acute and long-term success

Manually guided RF ablation for PV isolation has emerged as a standard second line treatment in patients with symptomatic AF [1]. Nevertheless, efforts continue to improve success rates and patient safety as well as procedural efficacy reducing procedure times and radiation exposure to patients and operators. 
Fig. 4 Procedural parameters. RMN-guided PV ablation was associated with longer procedure duration (left, upper panel), longer ablation times (right, upper panel), and longer RF current application duration (left, lower panel). In contrast, fluoroscopy time was lower in the RMN than in the CON group (right, lower panel)
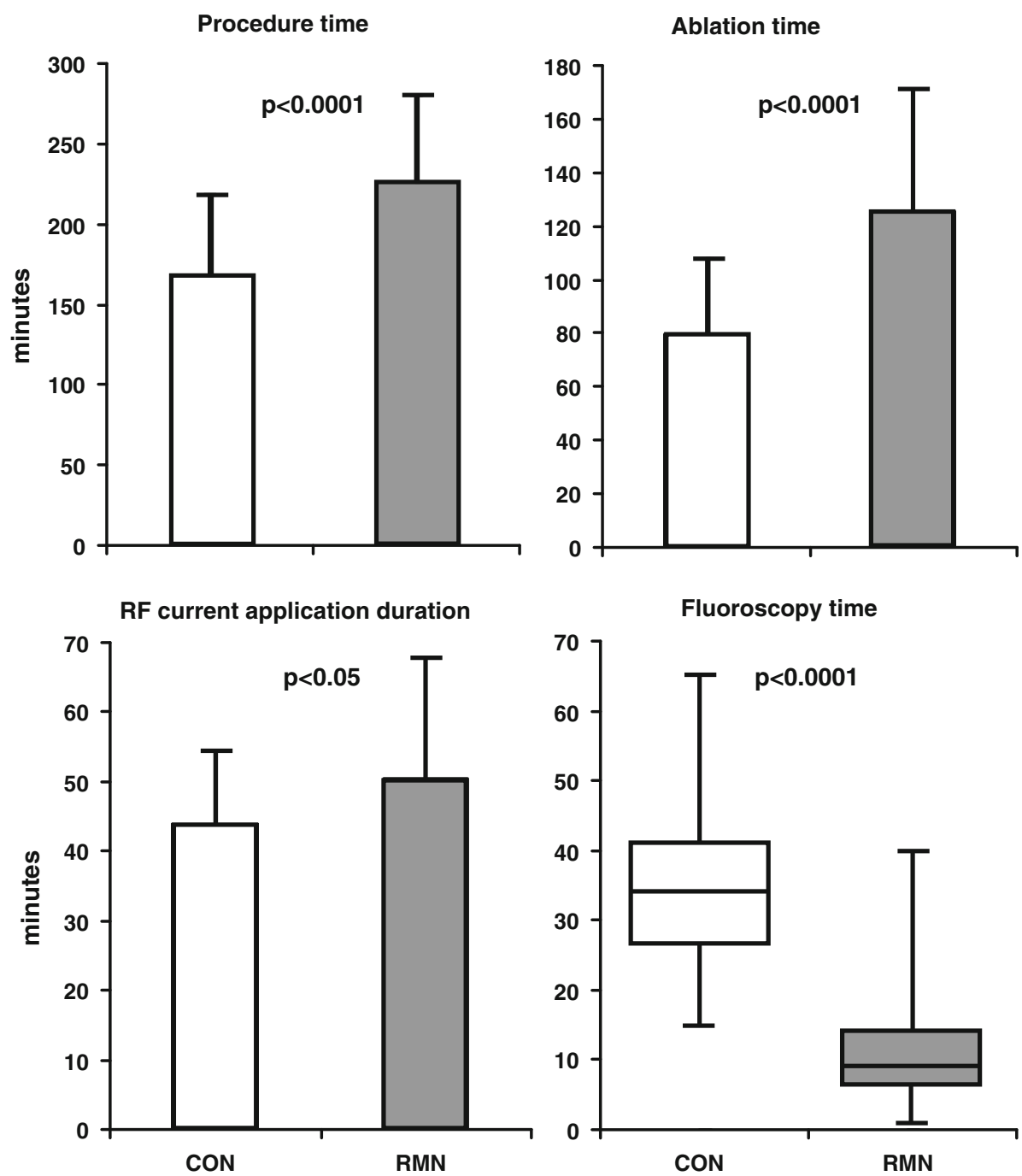

In our analysis, acute isolation of all PVs and long-term freedom from symptomatic $\mathrm{AF}$ or any $\mathrm{AF} / \mathrm{AT}$ was achieved in a comparable proportion of patients in the RMN and the CON group. So far, only limited data are available on the clinical utility of the RMN system for catheter-based PV isolation [5-10]. Early reports used nonirrigated ablation catheters, which limits the comparability to our results $[7,8,10]$. Very recently, first reports on the efficacy of RMN-guided PV isolation with an open-irrigated ablation catheter have been published by single centers [5, 6, 9]. Chun et al. [6] evaluated the efficacy of an RMN-guided open-irrigated ablation catheter in a premarket design and compared it to the efficacy of the advanced, marked-released RMN catheter. With the currently available catheter, the group achieved isolation of all PVs in $93 \%$ of the cases, and $70 \%$ of the patients remained in sinus rhythm during a median follow-up of 18 months. Arya et al. [5] report in their retrospective analysis complete isolation of all PVs during RMN-guided PV ablation with an open-irrigated catheter in $88 \%$ of the cases. Sixty- one percent of the RMN patients as compared to $68 \%$ in the conventional group remained free from AF after 6 months of follow-up. The retrospective analysis by Miyazaki et al. [9] demonstrated acute isolation of all 4 PVs in $87 \%$ of the 30 patients investigated with an RMN-guided open-irrigated catheter. At 12 months of follow-up after a single procedure, $69 \%$ of the patients were free of $\mathrm{AF}$ recurrence without antiarrhythmic drugs as compared to $62 \%$ in the manual group.

In the present study, long-term freedom from symptomatic AF was achieved in $83 \%$ of all patients with $1.5 \pm 0.6 \mathrm{PV}$ isolations. This data is in line with the general success rate from a recent meta-analysis from Cappato et al. [3], who reported that $85 \%$ of the patients were free from symptomatic AF after a median of 1.4 procedures. The long-term freedom from any AT/AF after a single procedure observed in our study is comparable to recently published data by Wilber et al. [12]. However, a meta-analysis by Calkins et al. [13] revealed a single procedure success rate of $72 \%$ (on or off antiarrhythmic 


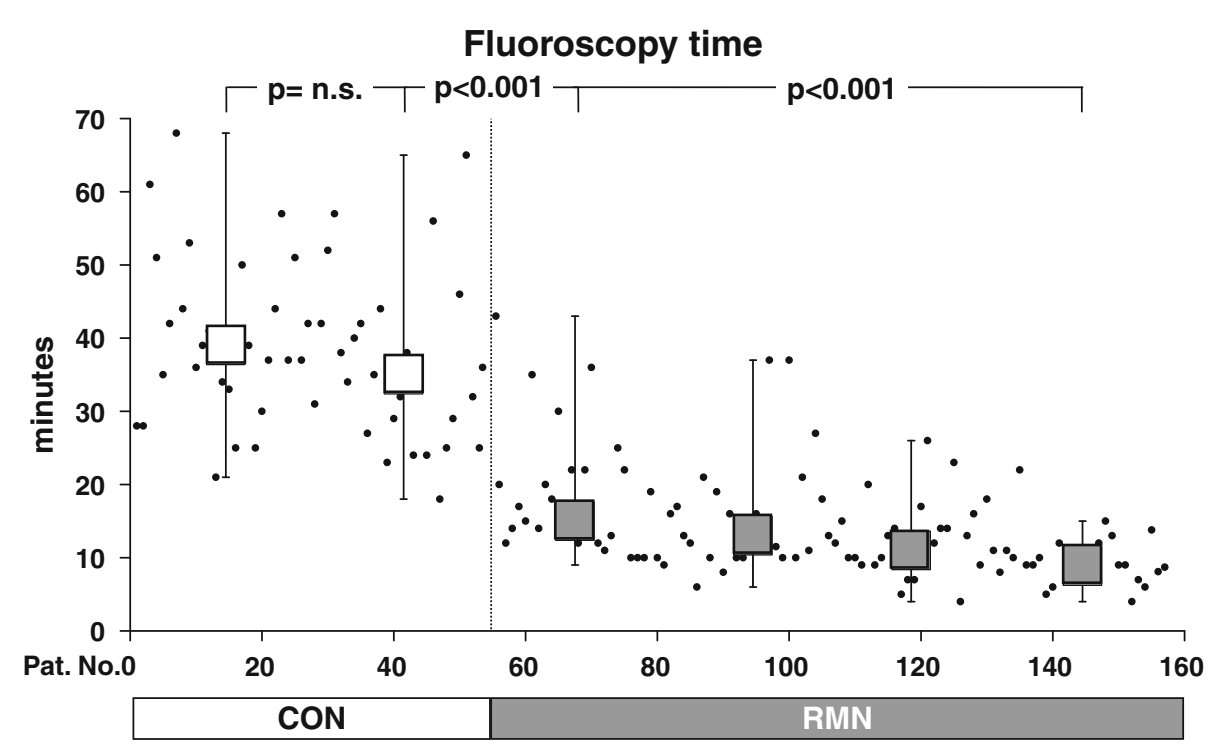

Fig. 5 Fluoroscopy time. The small dots represent the single cases. Patients were grouped into bins of 27 patients. The boxes and whiskers represent the median, minimum and maximum of the respective group. The $\mathrm{CON}$ group consists of the first 54 patients. The RMN group consists of the following 103 patients (Patient 55 to 157,

medication). The lower procedure success rate in our study as compared to this meta-analysis and the study by Chun et al [6] and Miyazaki et al. [9] may have several explanations. First, a larger proportion of patients in our study had enlarged left atria or presented with persistent AF. Several prior investigations as well as our own data indicate that these factors may influence the success of AF ablation [3, 14-16]. Second, we performed PV isolation without additional ablation also in patients suffering from persistent AF. In these subjects, PV isolation combined with linear lesions (e.g., mitral isthmus line) and/or ablation of complex fractionated electrograms may increase the success rate of the procedure [17]. This may potentially occur at the cost of an increased incidence of atrial tachycardias $[18,19]$ and other procedural complications [2, 20]. At our institution, we therefore prefer an approach to combine the effects of circumferential PV isolation with antiarrhythmic medication as needed. This concept can explain the higher proportion of patients on antiarrhythmic drugs in our study but is supported by the relatively low incidence of major complications. Third, we verified electrical isolation of the PVs without the aid of a circular mapping catheter. Although the use of a circular mapping catheter is still considered as the gold standard to ensure complete PV isolation, growing evidence exists [21-23] that a single-catheter approach (as utilized also in our study) may be equally effective for the achievement and verification of PV isolation [21, 22] and for the prevention of $\mathrm{AF}$ recurrences during follow-up [23]. We decided to apply the single-catheter approach for
4 values missing). Comparison of the first $27 \mathrm{CON}$ patients to the last $27 \mathrm{CON}$ patients revealed no significantly different fluoroscopy time. Fluoroscopy time significantly decreased from the last group of CON patients to the first group of RMN patients, and furthermore in the RMN group over time

mapping and ablation since the use of a manually guided circular mapping catheter increases the complexity of the procedure and contradicts the concept of remote navigation.

\section{Fluoroscopy time}

The use of RMN in the present investigation was associated with considerably shorter fluoroscopy times as compared to the CON approach, despite longer ablation times. In the RMN group fluoroscopy time decreased significantly over the course of the study. This benefit of RMN-guided ablation has previously also been observed by our group [24] and other investigators $[6,8,25]$ in studies on different arrhythmias including AF. The reduction in fluoroscopy time associated with RMN-guided ablation may be explained by the fact that the risk of cardiac perforation is rather low with the flexible tip of the magnetic catheter tip as compared to the stiff tip of a conventional ablation catheter tip. Large catheter movements without frequent fluoroscopic visualization of the catheter tip can therefore be made safely only with the flexible tip of the RMN ablation catheter. In this context, it appears noteworthy that two cardiac tamponades occurred in the RMN group of our study. The sub-acute time course of these complications lets one speculate that repetitive ablation at the perforation site rather than mechanical perforation of the LA wall accounts for these complications. The RMN system may increase catheter stability and thereby local lesion size [25-27]. We therefore rigorously avoided prolonged 
RMN-guided RF current delivery at single locations after the cases of sub-acute pericardial effusion.

\section{RF current delivery}

Our data indicate that more RF current has to be delivered when utilizing RMN-guided ablation as compared to the manual approach to achieve equally effective ablation lesions. This finding is in line with prior reports that suggest that RMN-guided ablation may produce less effective linear lesions $[7,24]$ but may be at least equally effective for focal ablation targets [25, 26]. The maximal endocardial force exerted by the RMN-system to the magnetic catheter tip is generally lower than that applied manually to the conventional ablation catheter tip [28]. Given the complex anatomy of the LA, one might assume that the tipto-tissue contact may be insufficient to produce effective ablation lesions with the RMN system in locations that are difficult to reach (e. g., right inferior PV) and/or at regions with thicker myocardium (e.g., the ridge between left atrial appendage and left superior PV). In return, exactly this feature of RMN may be desirable for increasing safety of the procedure. In this study, the magnetic field strength used was 0.08 Tesla. Since recently, the magnetic navigation system can apply higher field strengths of 0.1 Telsa, which might enhance the effectiveness of the produced lesions.

\section{Procedure duration and ablation time}

Longer ablation times with RMN-guided ablation also translated into longer procedure times as compared to the CON group of our study. These are not necessarily explained by the longer RF application time, which amounted to $7 \mathrm{~min}$ on average. In addition, the preparation time before the start of mapping contributes in a minor fashion as we demonstrated earlier for RMN-guided ablation of typical atrial flutter [24]. Rather an inherently slower speed in navigation of the LA or other cardiac chambers with RMN guided navigation as compared to manual navigation is reflected. When considering the numerous changes of catheter position necessary during the procedure, small delays between the computerized demand of vector direction change, movement of the permanent magnets and the magnetic field, and actual catheter movement must add to longer procedure times with RMNguided circumferential PV isolation.

\section{Limitations}

The main limitation is the non-randomized design of our study. Furthermore, inherent to AF studies asymptomatic episodes of AF may have been missed.

\section{Conclusion}

RMN-guided circumferential PV ablation with an openirrigated catheter provides comparable acute and long-term success rates as compared to manual catheter navigation. Complication rates of the procedure are similar between the two groups. The use of RMN is associated with markedly reduced fluoroscopy time, but prolonged ablation and procedure duration.

Acknowledgments The authors thank Klaus Jung for his assistance.

Conflict of Interest LL has received an educational and travel grant as well as a lecture fee from Stereotaxis Inc. DV and MZ have received previous grant support from Stereotaxis Inc. JS, MD, CS and GH: none.

Open Access This article is distributed under the terms of the Creative Commons Attribution Noncommercial License which permits any noncommercial use, distribution, and reproduction in any medium, provided the original author(s) and source are credited.

\section{References}

1. Fuster V, Ryden L, Cannom D et al (2006) ACC/AHA/ESC 2006 guidelines for the management of patients with atrial fibrillation: a report of the american college of cardiology/american heart association task force on practice guidelines and the european society of cardiology committee for practice guidelines (writing committee to revise the 2001 guidelines for the management of patients with atrial fibrillation): Developed in collaboration with the european heart rhythm association and the heart rhythm society. Circulation 114:e257-e354

2. Willems S, Drewitz I, Steven D, Hoffmann B, Meinertz T, Rostock T (2010) Interventional therapy of atrial fibrillation: possibilities and limitations. Dtsch Med Wochenschr 135:S48-S54

3. Cappato R, Calkins H, Chen S et al (2010) Ablation for human atrial fibrillation. Updated worldwide survey on the methods, efficacy, and safety of catheter. Circ Arrhythm Electrophysiol 3:32-38

4. Ferguson J, Helms A, Mangrum J et al (2009) Catheter ablation of atrial fibrillation without fluoroscopy using intracardiac echocardiography and electroanatomic mapping. Circ Arrhythm Electrophysiol 2:611-619

5. Arya A, Zaker-Shahrak R, Sommer P et al (2011) Catheter ablation of atrial fibrillation using remote magnetic catheter navigation: a case-control study. Europace 13:45-50

6. Chun K, Wissner E, Koektuerk B et al (2010) Remote-controlled magnetic pulmonary vein isolation utilizing a new irrigated tip catheter in patients with atrial fibrillation. Circ Arrhythm Electrophysiol 3:458-464

7. Di Biase L, Fahmy T, Patel D et al (2007) Remote magnetic navigation: human experience in pulmonary vein ablation. $\mathrm{J}$ Am Coll Cardiol 50:868-874

8. Katsiyiannis W, Melby D, Matelski J, Ervin V, Laverence K, Gornick C (2008) Feasibility and safety of remote-controlled magnetic navigation for ablation of atrial fibrillation. Am $\mathrm{J}$ Cardiol 102:1674-1676

9. Miyazaki S, Shah A, Xhaët O et al (2010) Remote magnetic navigation with irrigated tip catheter for ablation of paroxysmal atrial fibrillation. Circ Arrhythm Electrophysiol 3:585-589 
10. Pappone C, Vicedomini G, Manguso F et al (2006) Robotic magnetic navigation for atrial fibrillation ablation. J Am Coll Cardiol 47:1390-1400

11. Camm A, Kirchhof P, Lip G et al (2010) Guidelines for the management of atrial fibrillation: the task force for the management of atrial fibrillation of the European Society of Cardiology (ESC). Eur Heart J 31:2369-2429

12. Wilber D, Pappone C, Neuzil P et al (2010) Comparison of antiarrhythmic drug therapy and radiofrequency catheter ablation in patients with paroxysmal atrial fibrillation: a randomized controlled trial. JAMA 303:333-340

13. Calkins H, Reynolds MR, Spector P et al (2009) Treatment of atrial fibrillation with antiarrhythmic drugs or radiofrequency ablation: two systematic literature reviews and meta-analyses. Circ Arrhythm Electrophysiol 2:349-361

14. Berruezo A, Tamborero D, Mont L et al (2007) Pre-procedural predictors of atrial fibrillation recurrence after circumferential pulmonary vein ablation. Eur Heart J 28:836-841

15. Pappone C, Manguso F, Vicedomini G et al (2004) Prevention of iatrogenic atrial tachycardia after ablation of atrial fibrillation: a prospective randomized study comparing circumferential pulmonary vein ablation with a modified approach. Circulation 110:3036-3042

16. Themistoclakis S, Schweikert R, Saliba W et al (2008) Clinical predictors and relationship between early and late atrial tachyarrhythmias after pulmonary vein antrum isolation. Heart Rhythm 5:679-685

17. Brooks AG, Stiles MK, Laborderie J et al (2010) Outcomes of long-standing persistent atrial fibrillation ablation: a systematic review. Heart Rhythm 7:835-846

18. Cappato R, Calkins H, Chen SA et al (2005) Worldwide survey on the methods, efficacy, and safety of catheter ablation for human atrial fibrillation. Circulation 111:1100-1105

19. Sawhney N, Anousheh R, Chen W, Feld G (2010) Circumferential pulmonary vein ablation with additional linear ablation results in an increased incidence of left atrial flutter compared with segmental pulmonary vein isolation as an initial approach to ablation of paroxysmal atrial fibrillation. Circ Arrhythm Electrophysiol 3:243-248

20. Jaïs P, Hocini M, Hsu L et al (2004) Technique and results of linear ablation at the mitral isthmus. Circulation 110:2996-3002

21. Augello G, Vicedomini G, Saviano M et al (2009) Pulmonary vein isolation after circumferential pulmonary vein ablation: comparison between Lasso and three-dimensional electroanatomical assessment of complete electrical disconnection. Heart Rhythm 6:1706-1713

22. Dong J, Liu X, Long D et al (2009) Single-catheter technique for pulmonary vein antrum isolation: is it sufficient to identify and close the residual gaps without a circular mapping catheter? J Cardiovasc Electrophysiol 20:273-279

23. Eitel C, Hindricks G, Sommer P et al (2010) Circumferential pulmonary vein isolation and linear left atrial ablation as a singlecatheter technique to achieve bidirectional conduction block: the pace-and-ablate approach. Heart Rhythm 7:157-164

24. Vollmann D, Lüthje L, Seegers J, Hasenfuss G, Zabel M (2009) Remote magnetic catheter navigation for cavotricuspid isthmus ablation in patients with common-type atrial flutter. Circ Arrhythm Electrophysiol 2:603-610

25. Wood M, Orlov M, Ramaswamy K, Haffajee C, Ellenbogen K (2008) Remote magnetic versus manual catheter navigation for ablation of supraventricular tachycardias: a randomized, multicenter trial. Pacing Clin Electrophysiol 31:1313-1321

26. Davis D, Tang A, Gollob M, Lemery R, Green M, Birnie D (2008) Remote magnetic navigation-assisted catheter ablation enhances catheter stability and ablation success with lower catheter temperatures. Pacing Clin Electrophysiol 31:893-898

27. Ernst S, Ouyang F, Linder C et al (2004) Modulation of the slow pathway in the presence of a persistent left superior caval vein using the novel magnetic navigation system Niobe. Europace 6:10-14

28. Faddis MN, Blume W, Finney J et al (2002) Novel, magnetically guided catheter for endocardial mapping and radiofrequency catheter ablation. Circulation 106:2980-2985 